\title{
Poster: Compressed Sensing Inspired Approaches for Path Reconstruction in Wireless Sensor Networks
}

\author{
Rui Liu Yao Liang Xiaoyang Zhong \\ Department of Computer and Information Science \\ Indiana University - Purdue University Indianapolis, Indiana, USA \\ \{liurui, yliang, xiaozhon\}@cs.iupui.edu
}

\begin{abstract}
In this work, we investigate routing dynamics in mobile ad hoc wireless sensor networks (WSNs), which is of great importance for network performance analysis, operation optimization, system maintenance, and network diagnosis. We study packet path recovery for data collection in multi-hop dynamic WSNs at the sink based on compressed sensing approach. We extend our previous outing topology recovery (RTR) approach and evaluate its performance in comparison with the recent CSPR. Our work provides insights into the understanding of the profound impacts of different compressed sensing inspired approaches on their respective path reconstruction performance, and the resource requirement on sensor nodes. The evaluation results show that RTR can significantly outperform CSPR in various WSN setups.
\end{abstract}

\section{Categories and Subject Descriptors}

C.2.1 [Computer-Communication Networks]: Network Architecture and Design - Wireless communication.

\section{Keywords}

Wireless sensor networks; routing topology; compressed sensing; path reconstruction.

\section{INTRODUCTION}

It is increasingly important to understand packet routing dynamics in multi-hop and large-scale WSNs for network analysis, operation optimization, system maintenance, and network diagnosis. While a simple way is to directly record individual forwarding node's ID along the route in each packet, it is not scalable and also not resource-efficient. To address this challenge, several novel approaches for path-reconstruction in WSNs have been proposed recently, including Multi-hop Network Tomography (MNT) [1], PathZip [2], Routing Topology Recovery (RTR) [3], Pathfinder [4], and Compressive Sensing based Path Reconstruction (CSPR) [5]. MNT and Pathfinder rely on anchor packets to take advantage of the inter-packet correlation to infer packet path. PathZip compresses the path information into a hash value carried by each packet, in which the computation complexity grows exponentially with the size of WSN and thus may suffer from its scalability issue. RTR and CSPR are inspired by compressed sensing (CS). Recently, authors of CSPR [5] showed that CSPR performed better than MNT and Pathfinder.

This work focuses on the CS inspired approaches for path reconstruction in WSNs. Compressed sensing is a breakthrough

Permission to make digital or hard copies of part or all of this work for personal or classroom use is granted without fee provided that copies are not made or distributed for profit or commercial advantage, and that copies bear this notice and the full citation on the first page. Copyrights for third-party components of this work must be honored. For all other uses, contact the owner/author(s). Copyright is held by the author/owner(s).

MobiHoc'15, June 22-25, 2015, Hangzhou, China.

ACM 978-1-4503-3489-1/15/06.

http://dx.doi.org/10.1145/2746285.2764863

Liu, R., Zhong, X., Liang, Y., \& He, J. (2015). Understanding Compressed Sensing Inspired Approaches for Path Reconstruction in Wireless Sensor Networks. In 2015 IEEE International Conference on Smart City/SocialCom/SustainCom (SmartCity) (pp. 562-567). https://doi.org/10.1109/SmartCity.2015.129 technique in information theory and signal processing, which enables to recover a sparse signal from a small number of measurements. We note that the formulations of the RTR [3] and the recent CSPR [5] are very different. In view of this, we intend to understand if the two different CS-based formulations would have any profound impacts on their respective performances, as well as their resource requirements on each sensor node. In our RTR approach, the concept of base topology is introduced for WSN routing for data gathering, defined as the superset of all possible routing topologies of the WSN. Given a WSN of size $n$, the total number of directed wireless links (due to asymmetry wireless channel property) in the link space associated to the WSN base topology should be $N=n(n-1)-(n-1)=(n-1)^{2}$, modeled as a directed acyclic graph $\mathrm{G}(\mathrm{V}, \mathrm{E})$ without those wireless links outgoing from the sink. Our RTR approach is based on the observation that a WSN routing path, in principle, can be represented as a sparse link vector, each element in which corresponds to a link in the WSN link space of dimension $N=(n$ $1)^{2}$. In contrast, a path in CSPR is represented as a sparse node vector whose element corresponds to a node in the WSN. Therefore, in CSPR, the path representation space has its dimension $N=n$ for a given WSN of size $n$.

We extend our original RTR approach by devising new decoding algorithms for both reliable and lossy WSNs. We then evaluate the extended RTR versus the CSPR via careful network simulations. We present not only the evaluation results, but also our insights and analysis.

\section{RTR}

RTR formulates the path recovery as a novel CS-inspired problem. Let matrix $\Phi=\left\{\varphi_{i, j}\right\} \quad(1 \leq i \leq n-1,1 \leq j \leq$ $\left.(n-1)^{2}\right)$ represent a routing matrix in a WSN of size $n$ (including the sink), whose elements $\varphi_{i, j}$ are defined as

$$
\varphi_{i, j}=\left\{\begin{array}{l}
1, \text { the ith path traverses over the jth link; } \\
0, \text { otherwise. }
\end{array}\right.
$$

Given a path measurement vector $Y$ at the WSN sink for a collection cycle, reconstruct the link vector $X$ and matrix $\Phi$, so that

$$
\widehat{X}=\arg \min \|X\|_{0} \text { subject to } Y=\widehat{\Phi} X,
$$

where $l_{0}$-norm $\|X\|_{0}$ is the number of nonzero elements in $X$.

The original RTR algorithms [3] assume very little information available for path reconstruction, and thus require that packets are received at the sink in sequence, which may not be always true in practice. We extend the original RTR by devising new decoding algorithms to overcome this limitation. We assume that source node ID, hop count, and source node's parent ID are available in 
each packet. However, as hop count and source node's parent node ID are already available in WSN routing protocols such as Collection Tree Protocol (CTP), the enhanced RTR will make use of this available information without adding new overhead to WSN packet.

Each packet carries an aggregated path measurement, which initially is set to zero. The measurement encodes the path information along its route towards the sink, updated at each intermediate forwarding node. With a small and fixed overhead, RTR in-network encoding operations are the modular sum and XOR of the carried measurement in the packet and the value of currently traversed link in the route. RTR computes the unique label value $l_{u, v}$ of each individual link $e(u, v)$ online using the simple labeling function given in [3], based on unique IDs of the two endpoint nodes $u$ and $v$. If a node's ID has 16 bits, a link value then is 32 bits. Thus, a path measurement will have 32 bits for each modular sum and XOR result, resulting in total eight bytes overhead per packet. Once a packet arrives at the sink, its routing path will be decoded by our devised algorithms using its aggregated path measurement.

\section{EVALUATIONS}

We conducted detailed lossy WSN simulations using TOSSIM, the standard network simulator in TinyOS. Two important WSN topologies, the line topology and the grid topology are considered. For line topology, the sink node sits at one end of the line; for grid topology, the sink node sits at a corner. The sink collects WSN sensor readings from all nodes every five minutes. Table I gives a summary of the statistics of the simulations.

We evaluate the performance of RTR versus CSPR on per-packet path reconstruction as well as on path group reconstruction. CSPR adopts a CS solver CoSaMP to recover a path vector with $M$ measurements, and requires that $k<N / 2$ and $M \geq 3 k$, with $k$ nonzero items in the path vector of dimension $N$; otherwise, it outputs "Fail". Fig. 1 shows the performance comparison of RTR versus CSPR approaches for line and grid topologies. In both topologies, RTR performs significantly better than CSPR in per-packet path recovery. For path group reconstruction, RTR outperforms CSPR even more drastically. The insights are as follows. In WSN line topology with $n$ nodes, for example, the sparsity requirement of CSPR (due to CoSaMP) will not be satisfied if the number of hops is equal or larger than $(n-2) / 2$. Hence, in the simulation with 15 nodes, all the path groups with hop counts equal or larger than 6 are unrecoverable by the CSPR because their node vectors are not sparse. It is found that only $16.58 \%$ of the path groups in line topology meet the sparsity property (hop count $\leq 6$ ), among which $76.29 \%$ cannot collect sufficient packets for path reconstruction. In summary, $96.07 \%$ and $98.07 \%$ of the path groups cannot be recovered by the CSPR for the line and grid WSN topology, respectively.

\section{CONCLUSIONS}

We devise new RTR path decoding algorithms, and compare the two CS-inspired WSN path reconstruction approaches RTR versus CSPR. The evaluation results profoundly reveal that due to the different problem formulations, the RTR and CSPR exhibit dramatic difference between their respective performances. In particular for path group reconstruction, the CSPR was only able to recover $3.93 \%$ and $1.93 \%$ of the total path groups for simulated line and grid WSNs respectively. In contrast, the RTR was able to recover $94.36 \%$ and $81.48 \%$ of the total path groups for line and grid topology WSNs respectively. We show that the undesirable performance of CSPR is mainly caused by two critical drawbacks in its formulation: (1) the path node vector would largely violate the sparsity requirement of CS solvers for linear WSNs and other similar WSN topologies; and (2) most of the highly dynamic paths may not occur so frequently that the CSPR could not collect the sufficient number of packets in those path groups to recover them even after a long collection time (e.g., more than 2100 data collection cycles).

Table 1. Simulation statistics

\begin{tabular}{|c|c|c|}
\hline WSN Topology (size) & Line (15) & Grid (225) \\
\hline Packet reception rate & $98.60 \%$ & $87.10 \%$ \\
\hline Total received packets & 29962 & 44855 \\
\hline Total collection cycles & 2173 & 230 \\
\hline Total path groups & 585 & 19380 \\
\hline Longest path (hops) & 14 & 27 \\
\hline
\end{tabular}

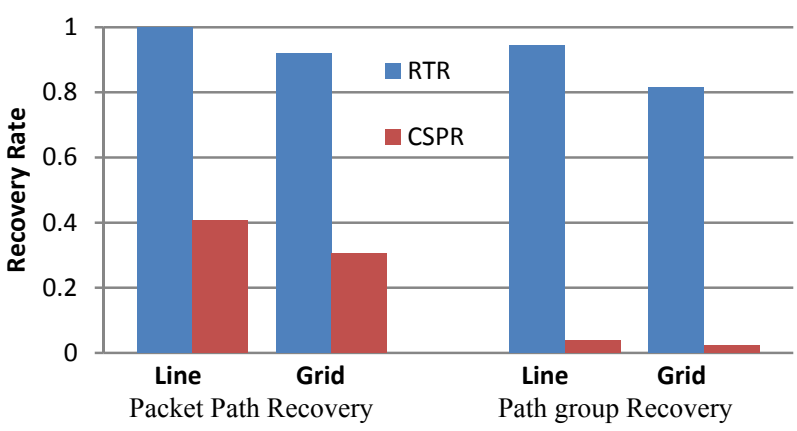

Figure 1. Performance evaluation of RTR vs. CSPR.

In addition, RTR uses much less resource for path encoding than CSPR. While both RTR and CSPR have the same packet overhead of eight bytes, CSPR requires 200 bytes in each node for storing its dictionary, whereas RTR needs none.

\section{ACKNOWLEDGMENTS}

This work was supported in part by NSF under CNS-1320132.

\section{REFERENCES}

[1] M. Keller, J. Beutel, and L. Thiele. How was your journey? Uncovering routing dynamics in deployed sensor networks with multi-hop network tomography. Proc. of SenSys, Nov. 2012.

[2] X. Lu, D. Dong, Y. Liu, X. Liao, and L. Shanshan. Pathzip: Packet path tracing in wireless sensor networks. Proc. of MASS, Oct. 2012.

[3] Y. Liang, and R. Liu. Routing Topology Inference for Wireless Sensor Networks. Computer Communication Review, Vol. 43, No. 2, pp. 22-27, April 2013.

[4] Y. Gao, W. Dong, C. Chen, J. Bu, G. Guan, X. Zhang, and $X$. Liu. Pathfinder: Robust path reconstruction in large scale sensor networks with lossy links. Proc. of ICNP, Oct. 2013.

[5] Z. Liu, Z. Li, M. Li, W. Xing, and D. Lu. Path reconstruction in dynamic wireless sensor networks using compressive sensing. Proc. of MobiHoc, Aug. 2014. 